\title{
Fatigue Crack Thermal Effect of Hydraulic Pipeline
}

\author{
Bowen Tang ${ }^{1, ~ a}$, Shaoping Wang ${ }^{1, b}$, Chao Zhang ${ }^{1, c}$, Shuo Zheng ${ }^{1 \mathrm{~d}}$ \\ ${ }^{1}$ School of Automation Science and Electrical Engineering \\ Beihang University, Beijing, 100083, China \\ atbw13941799668@126.com, bshaopingwang@vip.sina.com, ccz@buaa.edu.cn, \\ artin_zs@163.com
}

\begin{abstract}
Keywords: fatigue crack; thermal effect; hydraulic pipeline; infrared thermography; plastic zone area
\end{abstract}

\begin{abstract}
Fluid and solid coupling of aircraft hydraulic system can cause pipeline crack, while it is difficult to monitor its crack in real time. This paper studies the fatigue thermal effect, obtains the regulation of the thermal distribution, and establishes the thermal modeling of fatigue crack propagation. Through designing a type of tensile fatigue tubular specimen with 1Cr18Ni9Ti stainless steel, this paper simulates the fatigue behavior of the specimen in high cycle fatigue test, monitors the superficial temperature evolution in the process of fatigue cracks propagation and verifies the effectiveness of proposed method with infrared thermal imaging.
\end{abstract}

\section{Introduction}

Aircraft hydraulic pipeline is an important part of the aircraft hydraulic system that provides the high pressure hydraulic power form hydraulic pump to actuation system. According to the statistics, more than $30 \%$ aircraft failure is related to the hydraulic system while the fatigue crack is one of the most dangerous failures of hydraulic system failures [1]. Fatigue fracture failure will lead to the leakage of hydraulic oil, and even cause the failure of the aircraft hydraulic system.

Hydraulic pipe is mainly made of metal materials. With the fatigue crack growth and propagation, large amount of heat is generated. Infrared thermal imaging technology is a high-speed . high-sensitivity and non-destructive method to investigate the fatigue thermal phenomenon of metal materials. Luong [2] found that the intrinsic dissipation which was produced by the plastic deformation caused the change of temperature. Cura et al [3] showed that the temperature evolution of many materials can be presented as three stages when stresses are above fatigue limit. Yang et al [4] observed five stages in the temperature evolution of reactor pressure vessel steels by cyclic loading. There are few researches about the fatigue crack thermal effect of aircraft hydraulic pipeline.

This paper designed specimens with 1Cr18Ni9Ti stainless steel material which is commonly used in aircraft hydraulic pipeline [5]. During the fatigue testing, three infrared cameras were used to $360^{\circ}$ monitor the superficial temperature evolution and temperature field distribution of the specimen in the process of fatigue cracks initiate and propagate. The experimental results indicate that the proposed relationship between the stage of crack propagate and the stage of superficial temperature evolution is content.

\section{Temperature evolution regulation under fatigue crack propagation}

\section{Temperature evolution mechanism.}

In the fatigue process, the mental materials will produce plastic deformation, wherein the plastic deformation produces a large amount of heat. Literature [6] showed that the temperature variation process due to material fatigue can be divided into three stages shown in Fig. 1, in which $\Delta T$ is the temperature rise. 


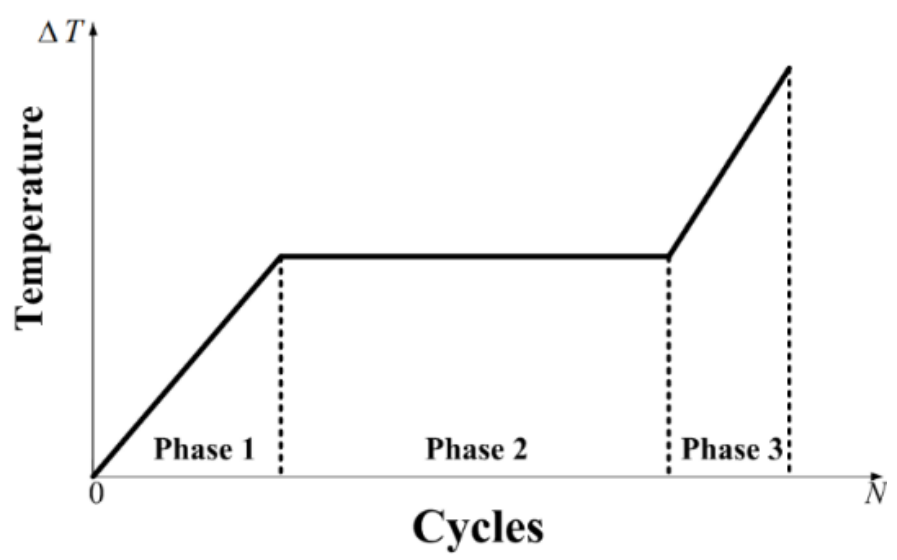

Fig. 1. Superficial temperature variation during fatigue propagation

There are three stages in superficial temperature evolution process, that is, the initial temperature increase, temperature stability and rapid temperature increase. The mechanical energy converts into heat in the fatigue process, mainly caused by the motion of material internal defection (such as dislocations, voids, etc.).

In the first stage, the number of mobile dislocations in the material is limited and the accumulated deformation energy changes little, so the mechanical energy converts into heat mostly that cause the temperature rise rapidly. In the second stage, the dislocation motion is more frequent and the accumulated deformation energy stored in each cycle is greatly increased compared with the first stage. In addition, the heat dissipation comes more due to the high temperature of the second stage. All that cause the rise rate of the time temperature curve to decrease [7]. In the third stage, the specimen fatigue fracture. In the end of second stage, the material has stored a lot accumulated deformation energy. Macroscopic crack initiate and propagate. The plastic deformation consumes mechanical energy, and $90 \%$ of that is converted into heat. As the speed of fatigue crack propagation is very fast, the temperature of the material increases rapidly.

Temperature evolution analysis of pipe fatigue crack.

The three stages superficial temperature evolution can be expressed by the energy balance theory. According to the energy balance theory, the variable quantity of the intrinsic energy equals to the quantity of external forces doing work and heat changes. The intrinsic energy includes the thermodynamic energy $U$, kinetic energy $K$, and some other forms of energy $E_{o t}$. The energy balance equation [8] can be expressed as:

$$
\dot{U}+\dot{K}+\dot{E}_{o t}=\dot{W}+\dot{Q}
$$

The specimens didn't move during the fatigue testing so $\dot{K}=0$. Neglect the changes of the other forms of energy so $E_{o t}=0$. So eq. (1) can be expressed as:

$$
\dot{U}=\dot{W}+\dot{Q}
$$

The thermodynamic energy $\dot{U}$ includes two parts. One is the internal kinetic energy changed $\dot{U}_{t}$ caused by the temperature change. The other is the accumulated deformation energy $\dot{E}_{s}$ caused by the microstructure changes of the material [9].

In the third stage, The plastic deformation of the crack tip consumes mechanical energy, and generates heat which leads to the temperature rise. In theory, the plastic deformation zone at the tip of the crack will appear significantly temperature rise and hotspots can be observed. According to the Von Mises yield criterion [10], the plastic zone of the crack tip is approximated circular, and the radius is 


$$
r(\theta)_{\text {planestress }}=\frac{1}{4 \pi}\left(\frac{K}{\sigma_{y s}}\right)^{2}\left(1+\frac{3}{2} \sin ^{2} \theta+\cos \theta\right)
$$

The crack in this experiment is a mode I crack, and the crack is simplified as a "natural crack specimen”, the curvature radius of the crack tip is close to zero $(\theta=0)$ :

$$
\begin{aligned}
r_{y s} & =\frac{1}{2 \pi} \frac{K^{2}}{\sigma_{y s}{ }^{2}} \\
K_{I} & =\sigma \sqrt{\pi a}
\end{aligned}
$$

Where: $r_{y s}$ is the radius of the crack tip plastic zone, $K$ is the stress intensity factor, $\sigma_{y s}$ is the yield strength of the material, $\sigma$ is the axial stress, $a$ is $1 / 2$ crack length

\section{Experimental verification}

\section{Test rig.}

\section{1)Specimens}

The specimens were made of 1Cr18Ni9Ti stainless steel material. In the center of the specimens, the outer diameter was $10 \mathrm{~mm}$ and the inner diameter was $8 \mathrm{~mm}$ (Fig 2). The chemical composition of this material was given in Table 1 . The mechanical properties of 1Cr18Ni9Ti stainless steel were listed in Table 2.

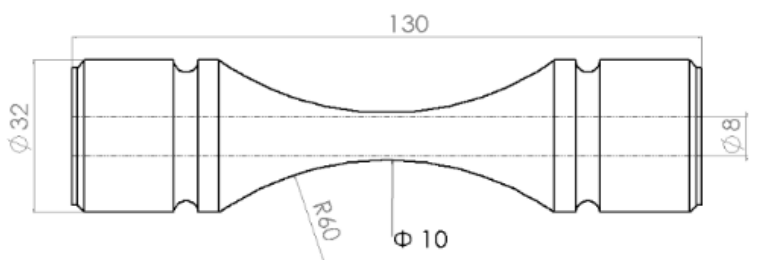

Fig. 2. Fatigue specimen

Table 1 Chemical composition of 1Cr18Ni9Ti stainless steel

\begin{tabular}{ccccccccc}
\hline Elements & $\mathrm{C}$ & $\mathrm{Si}$ & $\mathrm{Mn}$ & $\mathrm{P}$ & $\mathrm{S}$ & $\mathrm{Ni}$ & $\mathrm{Cr}$ & $\mathrm{Ti}$ \\
\hline Mass fraction/\% & $\leq 0.12$ & $\leq 1.00$ & $\leq 2.00$ & $\leq 0.035$ & $\leq 0.03$ & $8-11.00$ & $17-19$ & $5(\mathrm{C}-0.02) \sim 0.80$ \\
\hline
\end{tabular}

Table 2 The mechanical properties of 1Cr18Ni9Ti stainless steel

Ultimate strength $\sigma_{b} / \mathrm{MPa}$

Yield strength $\sigma_{s} / \mathrm{MPa}$

Elongation percentage $\delta^{\prime} / \%$

$\geq_{550} \geq_{205} \quad 40$

Before the test, the specimens have been polished with emery paper (30,240 mesh successively) to remove superficial oxide and covered with IR950 blackbody paint layer in order to increase thermal radiation rate.

\section{2)Experiment equipment}

The fatigue test machine was Swiss AMSLER high-frequency electromagnetic resonance fatigue test machine. The infrared thermal imaging camera used a FLIR Tau core with a $324 \times 256$ pixels and a temperature sensitivity better than $0.05^{\circ} \mathrm{C}$ at $30^{\circ} \mathrm{C}$. The spatial resolution was $1.316 \mathrm{mrad}$. Three infrared cameras were used to $360^{\circ}$ monitor the fatigue test. The fatigue tests were carried out at normal room temperature. 


\section{Test results.}

The fatigue tests were carried out twice at a temperature of $28{ }^{\circ} \mathrm{C}$ with the stress ratio $\mathrm{R}=0$ at a frequency $\mathrm{f}=195-197 \mathrm{~Hz}$. The specimen underwent $2.28 \times 10^{5}$ cycles fatigue loading at a stress level of $400 \mathrm{MPa}$ and underwent $3.07 \times 10^{5}$ cycles $390 \mathrm{MPa}$.

1) Temperature evolution during fatigue testing

Analyze the temperature evolution and the initial temperature increase details(fig.3).

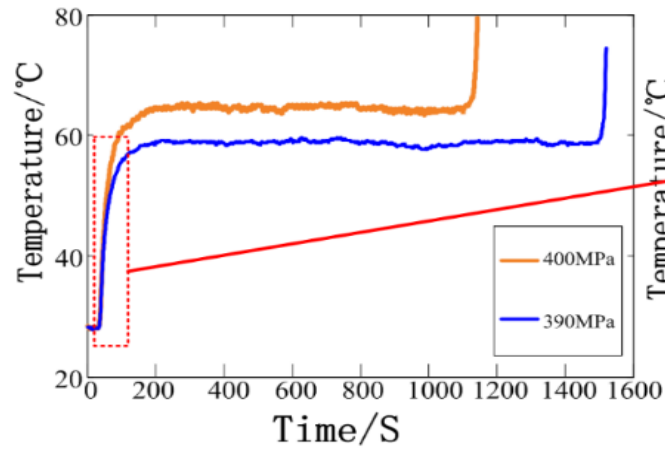

(a)

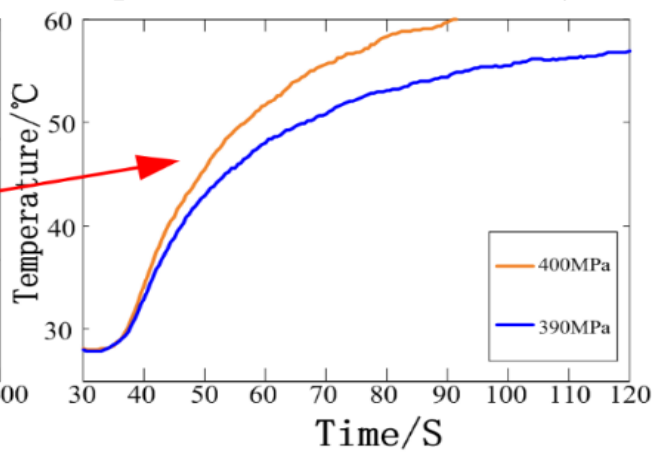

(b)

Fig. 3. Temperature evolution under different fatigue loading and detail view

Fig 3 shows a typical temperature evolution for a high-cycle fatigue test. Three stages of superficial temperature evolution were observed: an initial temperature increase (first stage), a temperature equilibrium (second stage), an abrupt temperature increase (third stage). The temperature rise of $400 \mathrm{MPa}$ was larger than 390MPa's and the temperature variation rate of 400MPa was larger than 390MPa's too.

2) Temperature field distribution during fatigue testing

Contrast the three groups of data under 400MPa loading (fig 4)

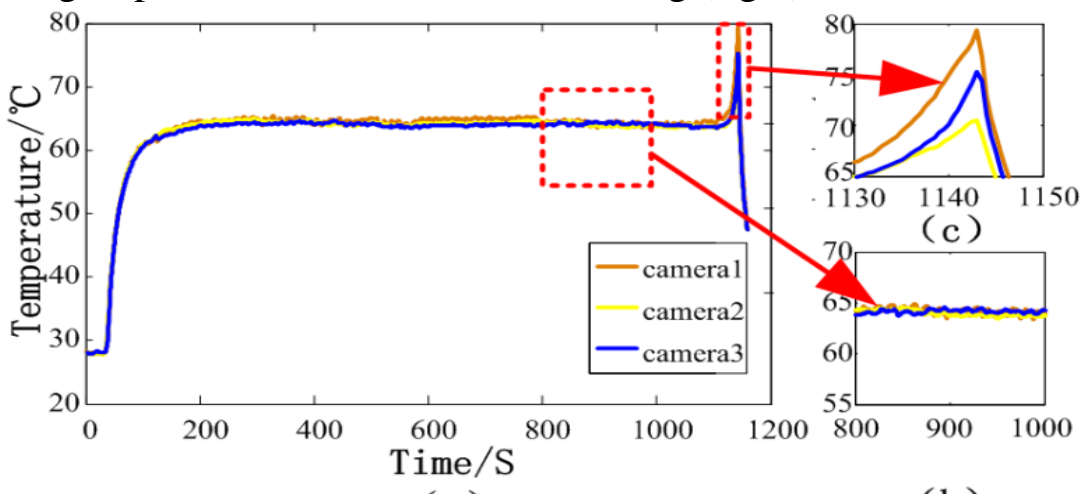

(a)

(b)

Fig. 4. Contrast the three groups of data under 400MPa loading

From fig 4(c), different areas in the center of the specimens have different temperature change in abrupt temperature increase stage. Contrast the infrared images in temperature equilibrium stage and abrupt temperature increase stage (fig 5)

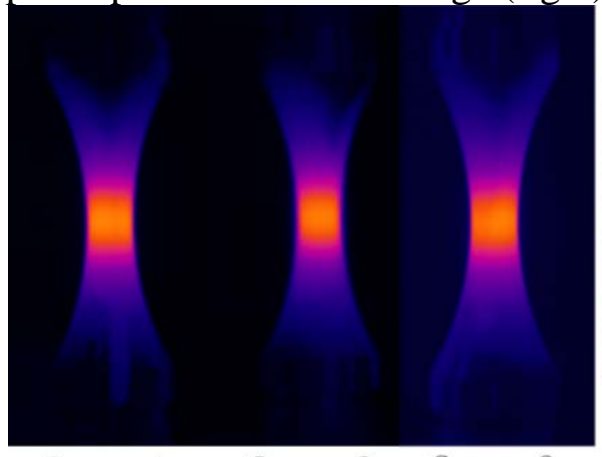

Cameral
Camera2

(a)

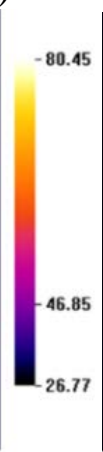

Camera3

$$
\text { Camera3 }
$$

ast the three

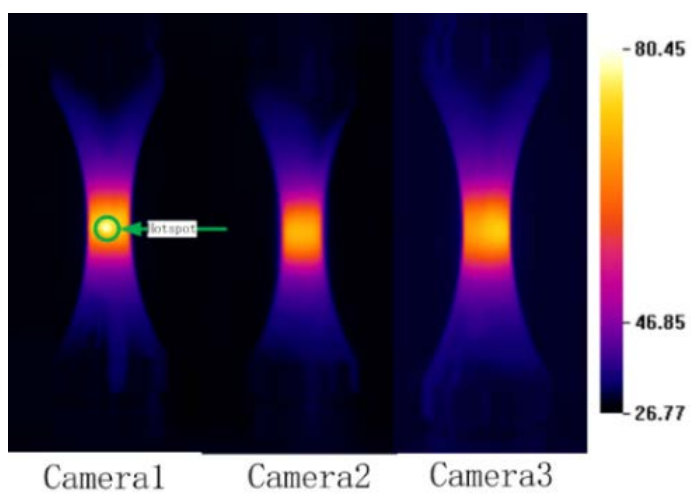

(b)

Fig. 5. Contrast the three groups of infrared thermal images 
From Fig. 5(a) and 5(b), only in the third stage, a hotspot was observed in the observation area of camera 1. Analyzing the specimen after the test, one of the crack tips appeared in the same position with the hotspot. It reported that high temperature region appears in abrupt temperature increase stage and fatigue crack appears in the same position where high temperature region appears.

\section{Discussion}

Analyzing the infrared thermal images, the highest temperature was $79.9^{\circ} \mathrm{C}$ and was recorded by camera 2 in 1536s. Order the images from camera 2 during fatigue crack propagation according time order.
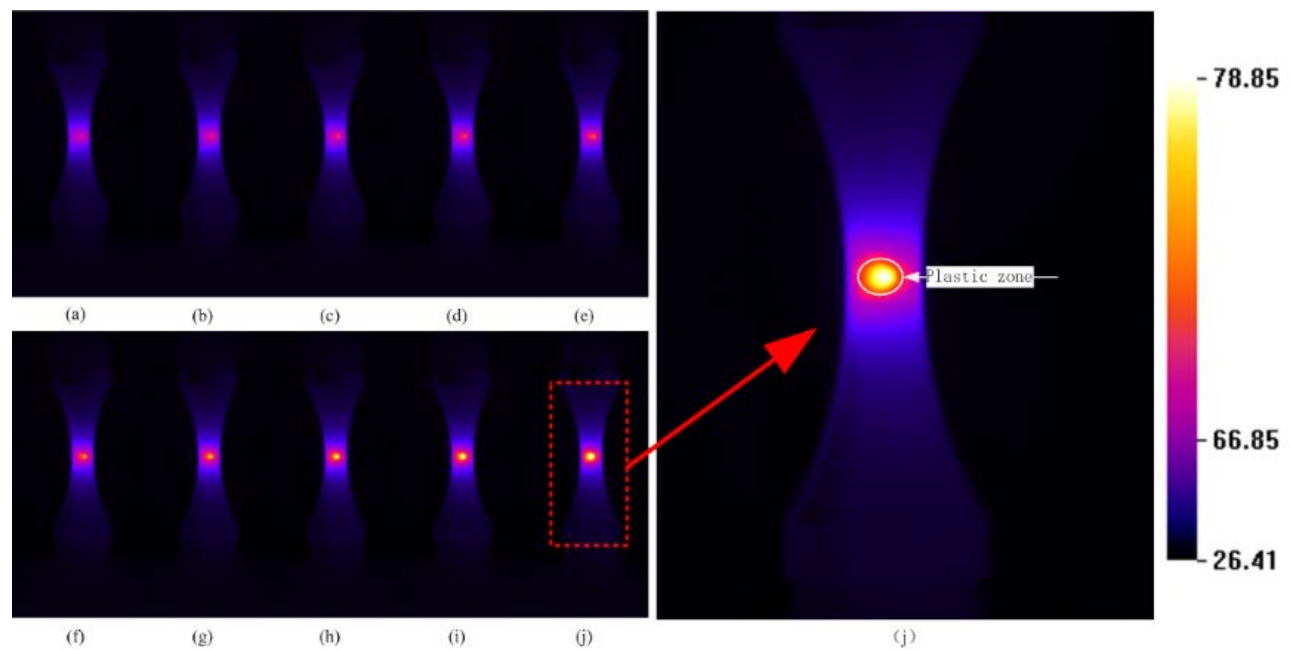

Fig. 6. Temperature field distribution during the fatigue crack propagation

From the fig.6 (a) to (J), the area of the highest temperature region (plastic zone) increased. The highest temperature region (plastic zone of the crack tip) is approximated circular. In the 390MPa test, the stress dropped to $355 \mathrm{MPa}$ at last, and the crack length came to $6 \mathrm{~mm}$ after test. After calculation, the crack tip plastic zone radius is $4.5 \mathrm{~mm}$, the area is about $63.6 \mathrm{~mm}^{2}$. The bright area shown in Figure 6(j) was the plastic zone of the crack tip. Measuring the plastic zone with a micrometer, the radius was approximately $7.9 \mathrm{~mm}$. So the area of crack tip plastic zone was about $65.2 \mathrm{~mm}^{2}$. The experimental result was compared with the theoretical value, the error was $2.45 \%$.

\section{Conclusions}

This paper utilizes infrared thermal imaging technology to monitor the fatigue crack propagation process, in which three stages of superficial temperature evolution were observed, viz. an initial temperature increase, a temperature equilibrium, an abrupt temperature increase.

The third stage of the temperature evolution was in agreement with the time of the macroscopic crack initiate and propagate. The temperature of the specimen was obviously increasing during the fracture process.

The highest temperature region of the specimen was in agreement with the macroscopic crack region.

Experimental results indicate that the macroscopic crack length was $6 \mathrm{~mm}$ after $3.07 \times 10^{5}$ cycles fatigue loading at a stress level of 390MPa. The theoretical value of the plastic zone area was about $63.6 \mathrm{~mm}^{2}$, the experimental result was about $65.2 \mathrm{~mm}^{2}$, the error was $2.45 \%$.

\section{References}

[1] Huazhi Wu, Deping Gao, Haiding Guo, A summary of the life characteristics of the probabilistic fatigue failure, Journal of Hubei Institute of Technology, 2002:18-22 (in Chinese).

[2] Luong M P. Nondestructive evaluation of fatigue limit of metals using infrared thermography. Nondestructive Characterization of Materials in Aging Systems. Mater Research Society, 
Warrendale, PA. 1998.275-288.

[3] Cura.F, Curti.G, Sesana.R. A new iteration method for the thermographic determination of fatigue limit in steels [J]. International Journal of Fatigue, 2005, 27: 453-459.

[4] B.Yang, P.K.Liaw, H.Wang, et al Thermographic investigation of the fatigue behavior of reactor pressure vessel steels [J]. Materials Science and Engineering A, 2001, 314: 131-139.

[5] Ledi Zhang, Xianyu Zhang, Study on dynamic characteristics and fatigue life estimation of aircraft hydraulic pipeline, Aviation Maitenance and Engineering, Vol.1, 2015:89-91

[6] L. Zhang, X.S. Liu, S.H. Wu, Z.Q. Ma, H.Y. Fang, Rapid determination of fatigue life based on temperature evolution[J]. International Journal of Fatigue,54(2013)1-6.

[7] Naderi M, Khonsari M M. A Thermodynamic Approach to Fatigue Damage Accumulation under Variable Loading[J]. Materials Science and Engineering: A, 2010, 527(23):6133-6139.

[8] G. Menegehetti, Analysis of the fatigue strength of a stainless steel based on the energy dissipation, International Journal of Fatigue 29 (2007) 81-94.

[9] G M.Analysis of the Fatigue Strength of a Stainless Steel Based on the Energy Dissipation[J]. International Journal of Fatigue, 2007, 29(1):81-94.

[10]Rang N, Palin-Luc T, Paris P C, et al. About the effect of plastic dissipation in heat at crack tip on the stress intensity factor under cyclic loading [J]. International Journal of Fatigue, 2013. 